Z. Klin. Chem. Klin. Biochem.

13. Jg. 1975, S. 137-142

\title{
Normalbereich des Gesamteiweißes und der Eiweißfraktionen des Liquor cerebrospinalis bei Kindern
}

\author{
Von $H$. D. Krause und $H$. Wisser
Aus dem Institut für Klinische Chemie (Geschäftsführender Direktor: Professor Dr. Dr. J. Büttner) der Medizinischen Hochschule Hannover

(Eingegangen am 10. Februar 1975)

\begin{abstract}
Zur Gewinnung von Normalwerten wurden an einer Stichprobe von 109 gesunden Kindern im Alter zwischen 0 und $13 \mathrm{Jahren}$ im Liquor cerebrospinalis das Gesamteiweiß, die verschiedenen Eiweißfraktionen nach elektrophoretischer Trennung und die Konzentration des IgG ermittelt. Das Gesamteiweiß wurde nach Fällung mit Trichloressigsäure mit der Biuret-Methode bestimmt. Die verschiedenen Fraktionen des Liquorproteins wurden nach Konzentrierung durch Druckfiltration, Auftrennung mit Hilfe der Mikrozonenelektrophorese auf Celluloseacetatfolie und Anfärbung mit Amidoschwarz 10 B photometrisch ermittelt. Die Bestimmung des IgG erfolgte mittels radialer Immunodiffusion.
\end{abstract}

\section{The normal range for total protein and protein fractions in the cerebrospinal fluid of children}

In order to establish normal values, samples of cerebrospinal fluid from 109 healthy children betwecn the ages of 0 and 13 years were analyzed for total protein, different protein fractions after electrophoretic separation, and IgG. Total protein was determined by the biuret method after precipitation with trichloroacetic acid. For the determination of the different protein fractions, the fluid was concentrated by high pressure filtration; the fractions were separated by microzone elektrophoresis on cellulose acetate membranes, then assayed photometrically after staining with amido black B. IgG was determined by radial immunodiffusion.

In einer früheren Publikation wurde über Normalwerte der Liquorelektrophorese auf Celluloseacetatfolie bei Erwachsenen berichtet (1). Der Anteil der Kinder an der damals untersuchten Stichprobe war zu klein, um eine eindeutige Aussage über Normalwerte machen zu können. Bei Durchsicht der Literatur fiel auf, daß Angaben über Normalwerte bei Kindern fehlen. Während unserer Untersuchung wurde von Siemes (2) über Normalwerte der Liquorelektrophorese bei Kindern berichtet. Allerdings handelt es sich hierbei um eine relativ kleine Stichprobe $(n=21)$, so daß es berechtigt erschien, das begonnene Projekt fortzusetzen und diese Ergebnisse unter Berücksichtigung von Alters- und Geschlechtsabunter Berücksichtigung von Alter und Geschlecht an einem größeren Kollektiv zu überprüfen.

Ein diagnostisch wichtiger Parameter der Liquorelektrophorese ist das $\gamma$-Globulin. Da IgA und IgM im Liquor von Normalpersonen mit den bisherigen Methoden nicht erfaßbar sind (3-6), sollte das IgG dem $\gamma$-Globulin der Elektrophorese entspreçhen. Es war somit naheliegend, ein Verfahren zu erproben, bei dem die Immunglobuline des Liquors direkt bestimmt werden können. Daher wurden diese in der gleichen Stichprobe mittels radialer Immunodiffusion bestimmt. IgA und IgM ließen sich auch bei der hier untersuchten Stichprobe nicht nachweisen.

\section{Methodik}

\section{Probengewinnung}

Die Liquorentnahme erfolgte bei allen Patienten durch Lumbalpunktion. Dic Proben wurden bei $+4{ }^{\circ} \mathrm{C}$ bis zur Analyse, die innerhalb von zwei Tagen erfolgte, aufbewahrt. Parallel zu jeder Liquorelektrophorese wurde eine Serumelektrophorese durchgeführt. Die Probe dazu wurde vor der Lumbalpunktion entnommen. Die für die Normalwerterstellung herangezogenen Ergebnisse wurden aus einem Kollektiv von Patienten der Hannoverschen Kinderheilanstalt ${ }^{1}$ ) ausgewählt. Die für den Ausschluß aus dem Gesamtkollektiv benützten Kriterien entsprachen denen an anderer Stelle schon publizierten (1). Wurde ein Patient mehrfach punktiert, wurde nur eine Liquoruntersuchung zur Normalwerterstellung benützt. In die Normalwertstudie konnten 109 Probanden ( 75 männliche und 34 weibliche) im Alter von 0 bis $13 \mathrm{Jahrcn}$ einbezogen werden.

Für die statistische Auswertung wurde die von uns untersuchte Stichprobe in 7 Altersklassen (AK) eingeteilt:

AK $1=0-0,5$ Jahre, AK $2=0,5-2$ Jahre, AK $3=2-4$ Jahre, AK 4 = 4-6 Jahre, AK 5 = 6-8 Jahre, AK 6=8-10 Jahre und AK $7=10-13$ Jahre.

\section{Bestim mungsverfahren}

Dic Gcsamteiweißbestimmung erfolgte nach Trichloressigsäurefällung mit der Biuret-Methode, die Bestimmung der Eiweißfraktionen nach Konzentricrung durch Druckfiltration mittels

1) Herrn Dr. Hamann und Herrn Priv. Doz. Dr. Natzschka danken wir für die Unterstützung der Arbeit durch Überlassung von Liquor- und Serumproben. 
elektrophoretischer Trennung und anschließender Anfärbung mit Amidoschwarz 10 B.

Hinsich tlich methodischer Einzelheiten sei auf vorangehende Publikationen $(1,7)$ verwiesen.

Die Bestimmung der Immunglobuline wurde mittels der einfachen radialen Immunodiffusion durchgeführt. Benützt wurden LC-Partigen-Platten der Firma Behring ${ }^{2}$ ). Die quantitative Auswertung erfolgte über Eichkurven, die durch Verdünnen eines Immunglobulinstandards gewonnen wurden. 48 Stunden nach Auftragen der Proben (Probevolumen $20 \mu$ l Nativliquor) wurden die Platten 24-48 Stunden mit $9 \mathrm{~g} / \mathrm{l} \mathrm{NaCl}$-Lösung gewaschen, um Begleitproteine zu entfernen. Anschließend wurden sie zur besseren Sichtbarmachung der Präzipitatringe mit $40 \mathrm{~g} / 1$ wäßriger Tanninlösung überschichtet und dann eine Stunde zur Entfernung der überschüssigen Tanninlösung in Wasser gelegt. Die Bestimmung der Durchmesser der Präzipitatringe erfolgte mit einem Meßlineal auf $0,1 \mathrm{~mm}$ genau.

\section{Ergebnisse und Diskussion}

Hinsichtlich der Reproduzierbarkeit der angewandten Methoden sei auf frühere Publikationen $(1,7)$ verwiesen.

Bei der statistischen Auswertung der 109 Probanden wurde zunächșt eine mögliche Geschlechtsabhängigkeit der Parameter geprüft. Dazu wurde in jeder Altersgruppe die Lage der Werte der männlichen mit der Lage der Werte der weiblichen Probanden verglichen (WilcoxonMann-Whitney-Test). Es wurde kein signifikanter Unterschied $(\alpha \leqslant 5 \%)$ gefunden. Somit konnten die weiteren Berechnungen gemeinsam für die männlichen und weiblichen Probanden durchgeführt werden.

Weiterhin wurde eine mögliche Altersabhängigkeit der Meßwerte geprüft. Beim Gesamteiweiß, dem IgG und dem $\gamma$-Globulin $(\mathrm{mg} / \mathrm{l})$ wurde eine statistisch signifikante Altersabhängigkeit gefunden, bei den Relativwerten der einzelnen Fraktionen der Liquorelektrophorese dagegen nicht (Kruskal-Wallis-Test, Tab. 1).

Tab. 1. K-Werte und approximative Testschwellen (KruskalWallis-Test) für die Prüfung der Altersabhängigkeit von Gesamteiweiß, der elektrophoretisch getrennten Fraktionen sowie des IgG.

\begin{tabular}{lcc}
\hline Bestandteil & $\mathrm{K}$ & $\begin{array}{c}\text { approximative } \\
\text { Testschwelle }\end{array}$ \\
\hline Gesamteiweiß & 26,5 & $0,02 \%$ \\
Präalbumin & 9,9 & $12,9 \%$ \\
Albumin & 2.45 & $87,4 \%$ \\
$\alpha_{1}$-Globulin & 5,2 & $51,2 \%$ \\
$\alpha_{2}$-Globulin & 1,3 & $97,0 \%$ \\
$\beta-+\tau$-Globulin & 3,3 & $77,2 \%$ \\
$\gamma$-Globulin & 8,7 & $19,2 \%$ \\
IgG & 21,9 & $0,13 \%$ \\
$\gamma$-Globulin (mg/l) & 12,0 & $6,1 \%$ \\
\hline
\end{tabular}

2) Behringwerke, Marburg/Lahn
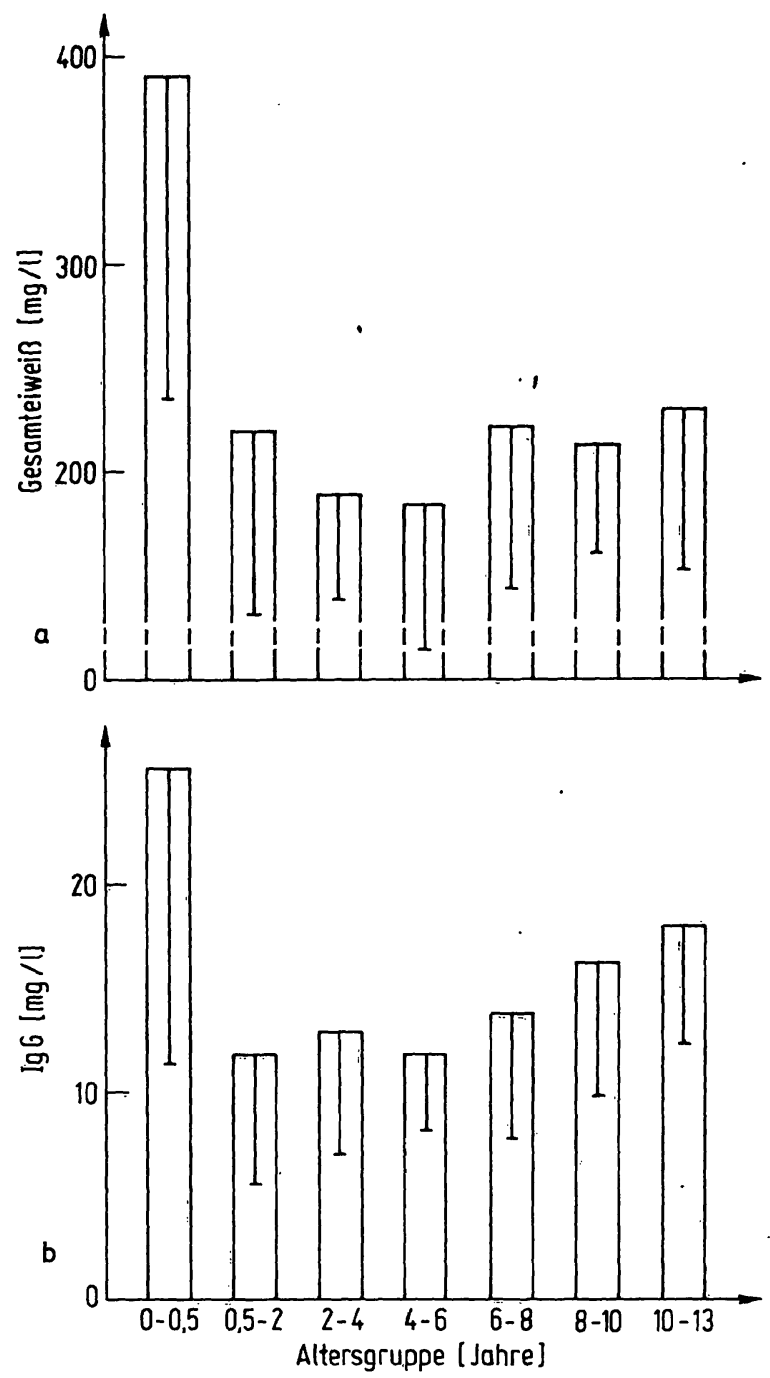

Abb. 1. Mittelwerte und Standardabweichungen von Gesamteiweiß (a) und IgG (b) des Liquor cerebrospinalis der verschiedenen Altersgruppen. (Werte aus Tabelle 2)

Diese Altersabhängigkeit der genannten Parameter ist insbesondere auf den Einfluß der Altersgruppe 1 zurückzuführen. Dies veranschaulicht Abbildung 1, in der die Mittelwerte und die einfachen Standardabweichungen von Gesamteiweiß und IgG der einzelnen Altersgruppen wiedergegeben sind.

Läßt man bei der Prüfung der Altersabhängigkeit die Werte der Altersklasse 1 (0-0,5 Jahre) weg, dann fallen die K-Werte für das Gesamteiweiß auf 5,3, für IgG auf 13,2 und für $\gamma$-Globulin (in $\mathrm{mg} / \mathrm{l}$ ) auf 6.5 , was approximativen Testschwellen von $38 \%, 2,2 \%$ und $26,1 \%$ entspricht. Für das Gesamteiweiß und das $\gamma$-Globulin (in $\mathrm{mg} / \mathrm{l}$ ) bestehen dann keine statistisch signifikanten Unterschiede mehr. Nach Weglassen der Altersgruppe 7 (10-13 Jahre) sinkt beim IgG der K-Wert auf 6,2, was einer approximativen Testschwelle von $18,2 \%$ entspricht. Wenn auch die statistische Auswertung für die Altersgruppen 2-7 (0,5-13 Jahre) keine statistisch signifi- 
kante Altersabhängigkeit zeigt, so läßt die graphische Darstellung (Abb. 1) jedoch nach dem starken Abfall der Werte gegenüber der Altersgruppe 1 eine ansteigende Tendenz der Mittelwerte für die Altersgruppen 2-7 erkennen. Die große Gesamtstreuung in den einzelnen Altersgruppen ist wahrscheinlich der Grund dafür, daß kein statistisch signifikanter Zusammenhang gefunden wurde. Dieser Befund wird durch die Ergebnisse eigener Untersuchungen (1) und von Pryce et al. (8) gestïtzt. Letztere fanden bei der Untersuchung eines Kollektivs von 35 männlichen und 32 weiblichen Probanden im Alter zwischen 0 und 90 Jahren eine signifikante Altersabhängigkeit der Gesamteiweißkonzentration, wenn auch diese nach der in der Arbeit wiedergegebenen graphischen Darstellung nicht sehr ausgeprägt ist. Die eigenen Untersuchungen (1) an 140 Probanden im Alter zwischen 16 und 74 Jahren zeigten nach Berechnung des Produkt-Moment-Korrelationskoeffizienten für das Gesamteiweiß in Abhängigkeit vom Alter einen statistisch signifikanten Zusammenhang. Außerdem lagen arithmetisches Mittel bzw. Median für diese Gesamtstichprobe mit $360 \mathrm{mg} / \mathrm{l}$ in der gleichen Größenordnung wie bei der Altersgruppe 1 der hier gezeigten Untersuchungsreihe, aber deutlich höher gegenüber den Werten der Altersgruppe 2 bis 7 (Tab. 2 - letzte Gruppe). Nach Altersgruppen getrennt und unter Berücksichtigung der an anderer Stelle gemachten Ausführungen über die Beschreibung eines Normalkollektives (1) sind die Ergebnisse für die 109 Probanden in Tabelle 2 zusammengefaßt.

Unter Berücksichtigung des Ergebnissẹ der statistischen Auswertung über die Altersabhängigkeit sind in der letzten Gruppe für das Gesamteiweiß die Altersgruppen 2-7, für die elektrophoretischen Fraktionen die Altersgruppen 1-7 und für das IgG die Altersgruppen 2-6 zusammengefaßt. Diese Zusammenfassung wurde aus praktischen Gründen gewählt, um eine vereinfachte Beurteilungsgrundlage zu haben. Sie ist auch aufgrund des Ergebnisses der statistischen Auswertung erlaubt.

Vergleicht man nun diese Werte mit denen von Siemes (2) an 21 Probanden, so fällt beim Gesamteiweiß die Gleichheit der Mittelwerte aus beiden Untersuchungsreihen auf, wenn auch die Streuung und damit der Normalbereich sich stärker unterscheiden. Allerdings benützte er als Auswahlkriterium für seine Stichprobe eine Eiweißkonzentration zwischen 100 und 300 mg/l. Die von Siemes mit $44 \mathrm{mg} / \mathrm{l}$ angegebene Streuung liegt deutlich unter der von uns ermittelten (Tabelle 2). Dies dürfte auf die Unterschiede in der Stichprobengröße und das recht willkürliche Auswahlkriterium bezüglich des Gesamteiweißes zurückzuführen sein. Von Nellhaus (5) wurden für das Gesamteiweiß (turbidimetrisch) an einer Stichprobe bei 73 Kindern folgende Werte ermittelt: Mittelwert $281 \mathrm{mg} / 1$, Standardabweichung $116 \mathrm{mg} / \mathrm{l}$, Bereich $50-700 \mathrm{mg} / \mathrm{l}$. Diese Werte zeigen gegenüber den in dieser Arbeit angegebenen relativ große Abwei- chungen. Ein Grund dafür dürfte in den unterschiedlichen Bestimmungsverfahren liegen.

Hinsichtlich der elektrophoretisch getrennten Fraktionen zeigen sich gegenüber dem Ergebnis der Untersuchung an Erwachsenen (1) mit Ausnahme des Präalbumins keine größeren Unterschiede. Vergleicht man die von Siemes (2) gewonnenen Werte mit denen in Tabelle 2 für die einzelnen Fraktionen, so zeigen sich auch hier ebenfalls beim Präalbumin, $\alpha_{1^{-}}, \alpha_{2}$ - und $\gamma$-Globulin größere Abweichungen. Diese Abweichungen sind möglicherweise durch Unterschiede im Einengverfahren bedingt, da gezeigt werden konnte, daß hier insbesondere beim Albumin und $\gamma$-Globulin Unterschiede auftreten können (7).

Wie aus Abbildung 1 ersichtlich, ist das IgG im Alter zwischen 0 und 0,5 Jahren hoch, um analog dem Gesamteiweiß auf niedrigere Werte abzufallen. Diese zunächst hohe Konzentration dürfte dadurch bedingt sein, daß der größte Teil des IgG des Liquor cerebrospinalis der Neugeborenenperiode, da es im Gegensatz zu IgM und IgA die Placenta passieren kann, von der Mutter stammt. Somit ist es erklärlich, daß die für diese Altersgruppe gewonnenen Werte denen von Erwachsenen (9) nahe kommen. Mit zunehmendem Alter zeigen Median und Mittelwert der jeweiligen Altersgruppe ansteigende Tendenz, wenn auch für die Altersgruppen 2, 3, 4, 5 und 6 kein statistisch signifikanter Unterschied festgestellt werden konnte. Bestätigt wird dies durch die oben erwähnte Untersuchung von Nellhaus (5), der einen Anstieg für den prozentualen Anteil des IgG am Gesamtprotein bis zum 8 . Lebensjahr fand. Allerdings war auch dieser Befund statistisch nicht signifikant. Das IgG wurde bei dieser Studie mittels Elektroimmunodiffusion bestimmt. Da im Liquor mit der verwendeten Methode kein IgA und IgM nachweisbar war, sollten IgG und $\gamma$-Globulin nach Umrechnung der Relativprozente über das Gesamteiweiß in Absolutwerte einander entsprechen. Es wurden daher die Produkt-MomentKorrelations-Koeffizienten für $\gamma$-Globulin $(\mathrm{mg} / \mathrm{l})$ in Abhängigkeit von den gemessenen IgG-Werten berechnet (Tab. 3).

Tab. 3. Produkt-Moment-Korrelations-Koeffižienten von IgG zu $\gamma$-Globulin (in $\mathrm{mg} / \mathrm{l}$ ).

\begin{tabular}{lll}
\hline Altersgruppe & Jahre & $\begin{array}{l}\text { Produkt-Moment- } \\
\text { Korrelations-Koeffizient }\end{array}$ \\
\hline 1 & $0-0,5$ & 0,8090 \\
2 & $0,5-2$ & 0,2993 \\
3 & $2-4$ & 0,9060 \\
4 & $4-6$ & 0,4584 \\
5 & $6-8$ & 0,5956 \\
6 & $8-10$ & 0,8189 \\
7 & $10-13$ & 0,8885 \\
$2-6$ & $0,5-10$ & 0,6388 \\
\hline
\end{tabular}




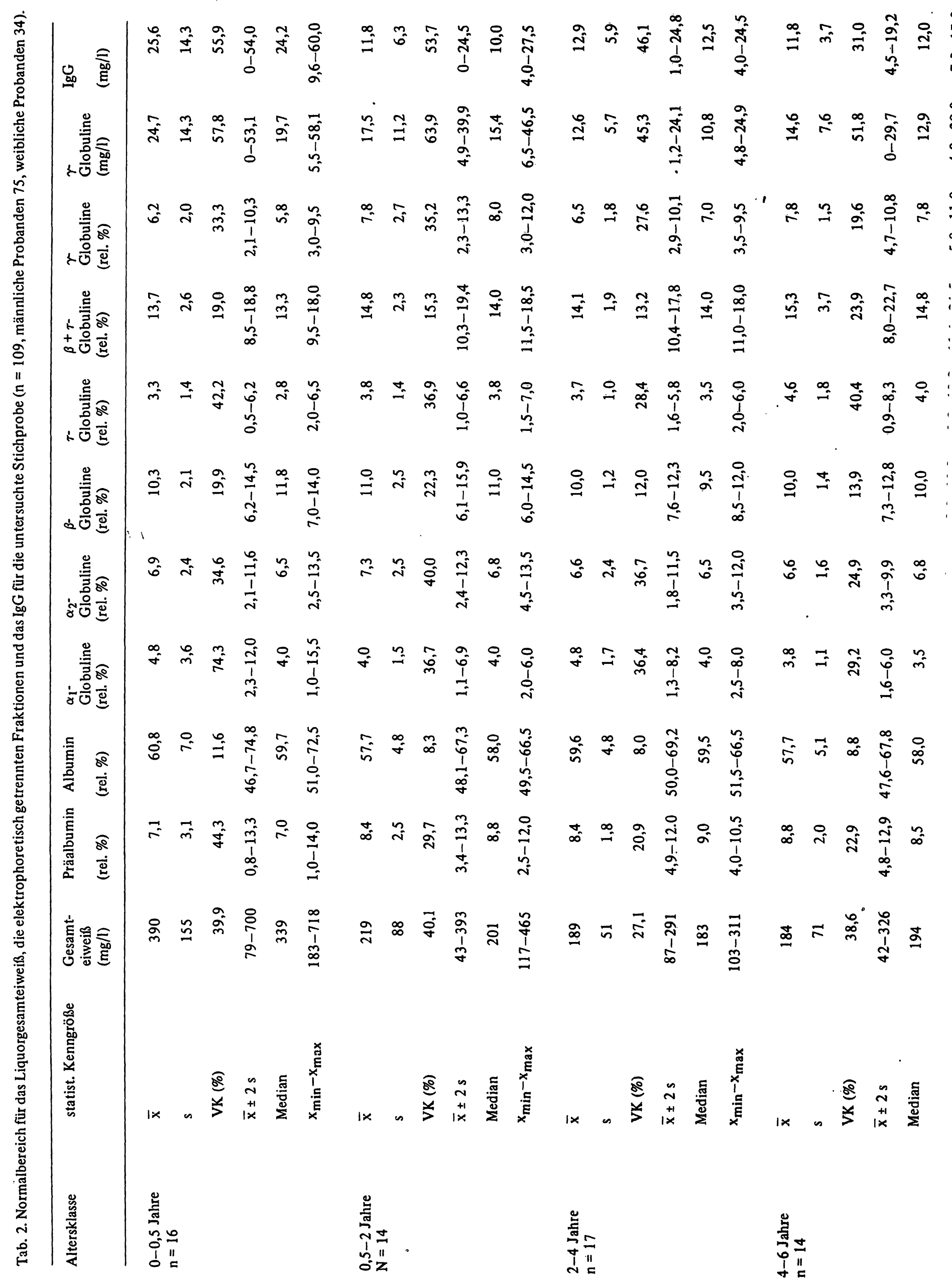




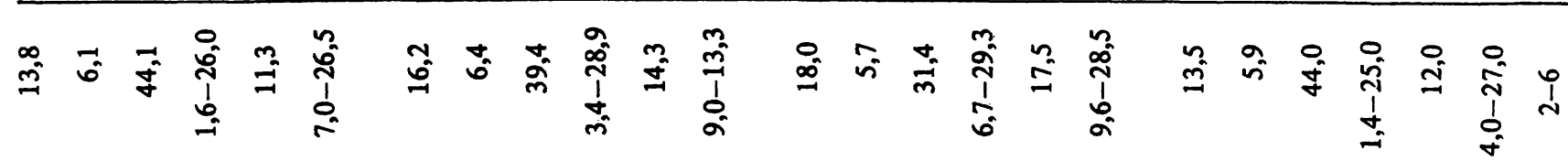

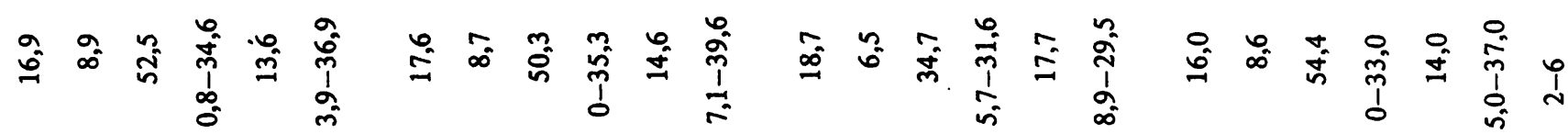

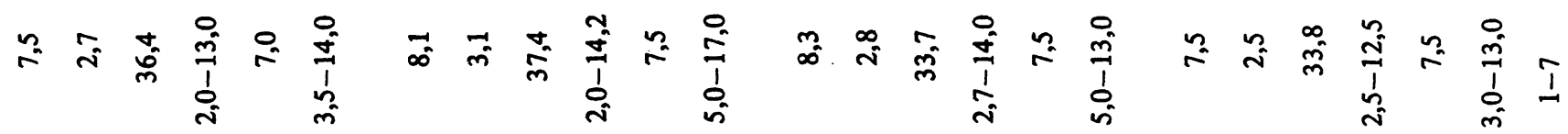

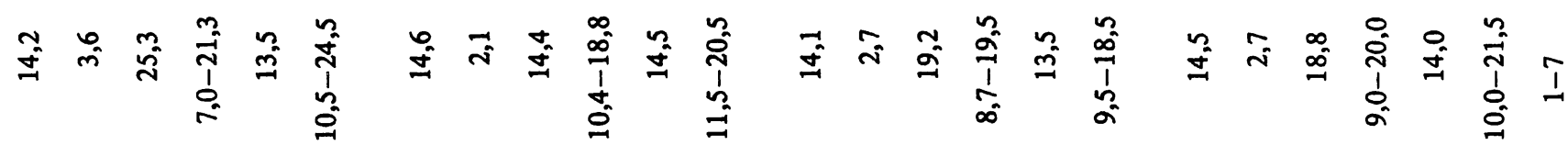

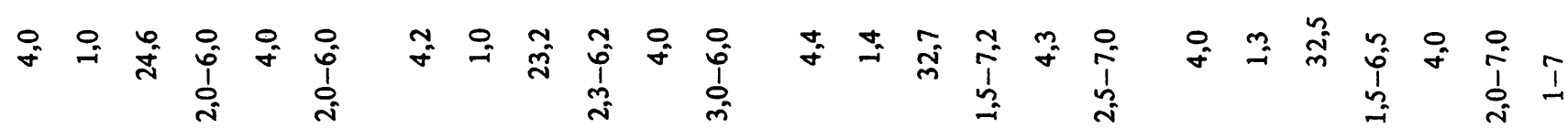

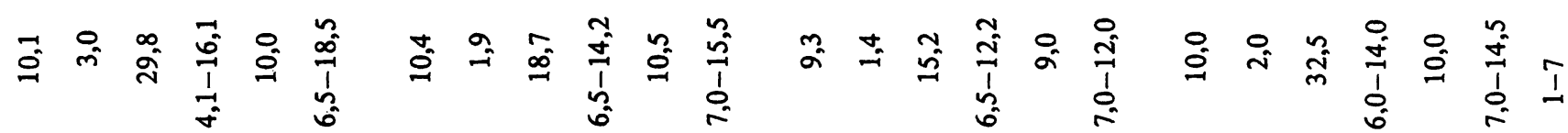

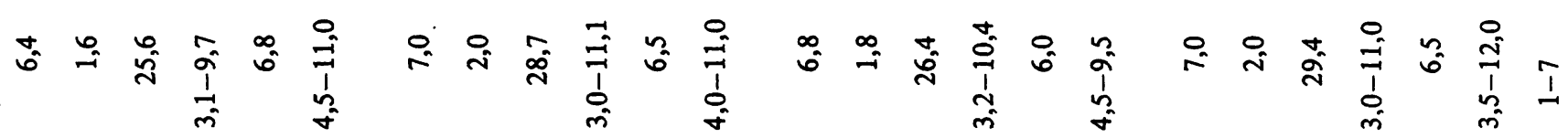

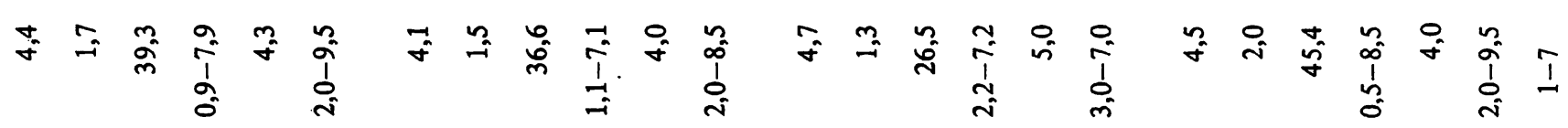

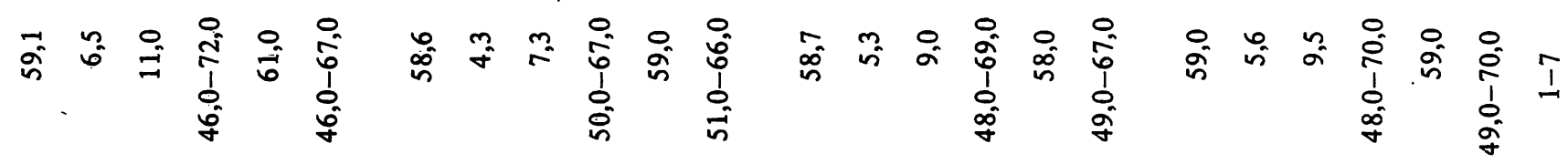

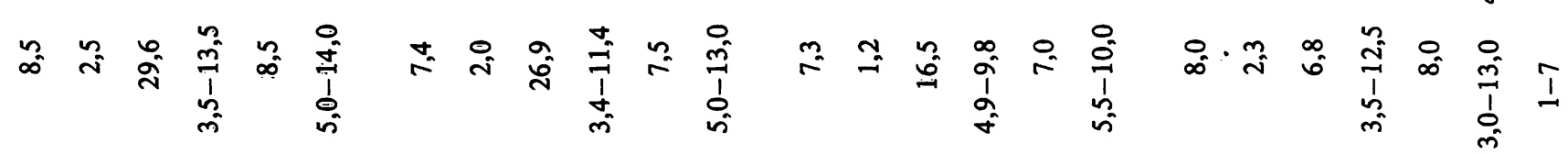

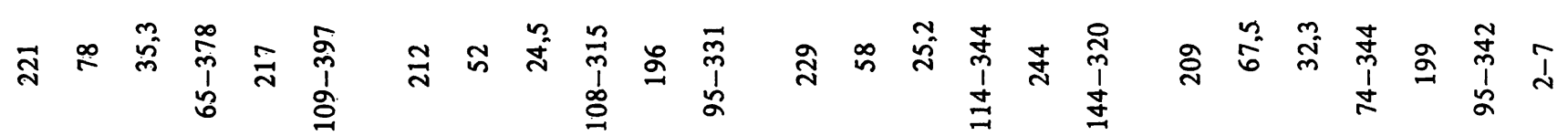

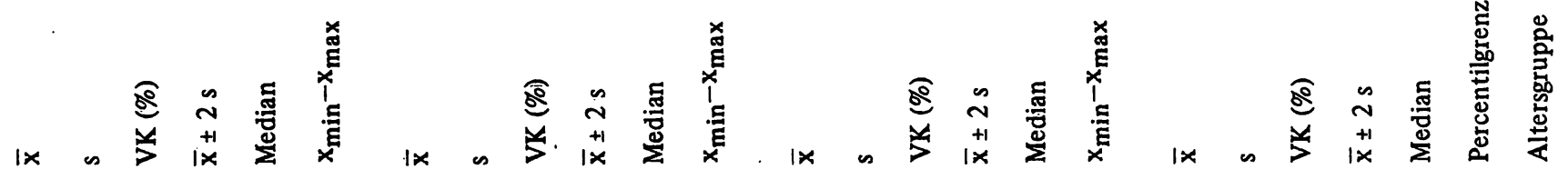

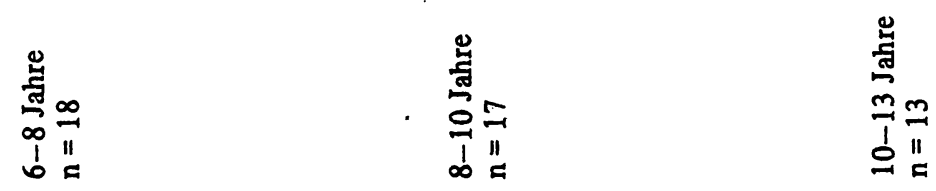

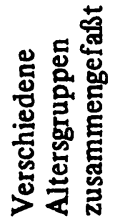


Es zeigt sich in den einzelnen Altersklassen eine unterschiedlich stark ausgeprägte Korrelation. Faßt man die Altersgruppen 2-6 zusammen, so besteht trotz unterschiedlicher Methodik und der sicherlich nicht sehr hohen Präzision beider Verfahren eine statistisch signifikante Korrelation $(\alpha \leq 5 \%)$.

\section{Literatur}

1. Mertin, J., Wisser, H. \& Doerr, P. (1971), diese Z. 9, 337-340.

2. Siemes, H. (1972), Mschr. Kinderheilk. 120, 262-264

3. Hartley, Th. F., Merill, Deborah A. \& Claman, H. C. (1966), Arch. Neurol. 15, 472-479.

4. Riddoch, D. \& Thompson, R. A. (1970), Brit. Med. J. I, 396-399

5. Nellhaus, G. (1971), Arch. Neurol. 24, 441-448.

\section{Danksagung}

Herrn Dr. E. Hansert, Leiter der Biometrischen Abteilung des Max-Planck-Institutes für Psychiatrie, München, danken wir für die Hilfe bei der statistischen Auswertung.

6. Sketh, N. K. (1971), J. Clin. Pathol. 24, 363-365.

7. Krause, H. D., Wisser, H. \& Pirke, K. M. (1975), diese Z. 13, 79-84.

8. Pryce, J. D., Gant, P. W. \& Saul, K. J. (1970), Clin. Chem. $16,562-565$.

9. Glasner, H. \& Wenig, Ch. (1973), Klin. Wochenschr. 51, 806-809

Priv. Doz. Dr. Dr. H. Wisser Abteilung für Klinische Chemie Robert-Bosch-Krankenhaus Stuttgart 